\title{
La vie des mots en analyse du travail
}

Words motion in work's analysis

La vida de las palabras en el análisis del trabajo

Muriel Henry et Maryse Bournel Bosson

\section{OpenEdition}

\section{Journals}

Édition électronique

URL : http://journals.openedition.org/activites/2002

DOI : $10.4000 /$ activites.2002

ISSN : 1765-2723

Éditeur

ARPACT - Association Recherches et Pratiques sur les ACTivités

Référence électronique

Muriel Henry et Maryse Bournel Bosson, "La vie des mots en analyse du travail », Activités [En ligne], 5-2 | octobre 2008, mis en ligne le 15 octobre 2008, consulté le 01 mai 2019. URL : http:// journals.openedition.org/activites/2002 ; DOI : 10.4000/activites.2002

\section{(c) (i) (9)}

Activités est mis à disposition selon les termes de la licence Creative Commons Attribution - Pas d'Utilisation Commerciale - Pas de Modification 4.0 International. 


\title{
La vie des mots en analyse du travail
}

\author{
Muriel Henry \\ CNAM. Centre de Recherche « Travail \& Développement » (CRTD) - Equipe Clinique de l'Activité (EA 4132) \\ 41, rue Gay-Lussac. 75005 Paris. \\ Mu.henry@orange.fr
}

\section{Maryse Bournel Bosson}

Université de Franche-Comté (EA 3188). 25000. Besançon.

Chercheur associé CRTD - Equipe Clinique de l'Activité (EA 4132)

marysebournelbosson@wanadoo.fr

\begin{abstract}
Words motion in work's analysis. Work analysis methods in "Clinic of Activity" involve professionals in confrontation with films of their real action. This method aims at creating development by opening up new ways of thinking, telling and acting in daily activity. Here, from datas got with such methods, about validation of experience work, we study the creative linguistic process by describing precisely results when searcher repetes professionnal's words.We use the concept of heterogeneousness, from Bakhtine, for understanding how words separate from meanings and things they were linked with, opening then new possible objectivations.
\end{abstract}

\section{KEYwORDS}

Crossed self-confrontation, clinic of activity, repetition, heterogeneousness

\section{Introduction}

Le point de départ de notre objet d'étude est un dispositif méthodologique visant à organiser la confrontation de membres de jurys en Validation des Acquis de l'Expérience (VAE) aux traces filmées de leur activité. L'objectif de cette confrontation basée sur l'instauration de dialogues entre professionnels à partir de leurs réalisations concrètes est de provoquer un développement individuel et collectif du «métier » ou plus exactement de l'activité. En tant que moyen d'action mobilisant le langage sur le travail, les méthodes d'autoconfrontations croisées (Clot, \& Faïta, 2000; Clot, Faïta, Fernandez, \& Scheller, 2001; Clot, 2005) cherchent avant tout à engager collectivement les professionnels à s'interroger sur ce qu'ils se voient faire. Le dispositif ainsi proposé rend possible le développement par les auteurs eux-mêmes des registres afférents aux gestes ou mots de l'activité de travail. Aux données issues de cette analyse de l'activité spécifique, nous attribuons une seconde vie, une autre destinée, celle de constituer une ressource pour produire des connaissances sur le fonctionnement du développement. En effet, si le dialogue réglé entre pairs sur les traces de leur activité est conçu comme le lieu privilégié de l'extension de la signification et de la production du sens ${ }^{1}$, il revient alors au chercheur, lorsqu'il dispose de ces données, de rendre compte, pour partie,

1. Les concepts de sens et de signification sont indissociables : la signification recèle un potentiel de sens que le dialogue réalise à chaque fois de manière singulière. Un modèle dynamique de la signification est à mobiliser: on se doit de distinguer la signification littérale, formelle, conventionnelle des mots et leur signification « réelle » qui s'affranchit de la première catégorie. Vygotski démontre que les significations des mots se développent à partir du double mouvement de la signification sociale et de l'expérience: «Le mot absorbe les contenus intellectuels et affectifs et se met à signifier plus ou moins que ce qui est contenu dans la signification. Plus parce que le cercle de ses significations s'élargit acquérant une série de zones chargées d'un nouveau contenu, moins parce que la signification abstraite du mot est rétrécie et limitée à ce que le mot signifie dans un seul contexte donné » (1934,p. 481). Mais Bakhtine va sans doute au-delà de la perspective vygotskienne, en ce qu'il introduit les « genres de discours » comme intercalaire 
des processus d'élaboration qui se réalisent pendant cette activité langagière ainsi que des conditions de sa mise en œuvre.

Dans l'espace de cet article, l'objet d'étude se limitera à analyser le déroulement de la première phase, celle de l'autoconfrontation simple. C'est en mobilisant la théorie du dialogisme telle qu'elle a été développée par Bakhtine, Volochinov et plus largement les travaux de linguistes s'inscrivant dans cette filiation (Authier-Revuz, 1995, 2000; Salazar-Orvig, 2000) que nous conduirons l'analyse. Celle-ci prend comme point de départ le constat plutôt intuitif d'un phénomène massif de ruptures dans le discours du professionnel commentant son activité de travail: phrases interrompues, silences, hésitations, recherche d'autres mots... On interroge cette non-coïncidence entre les mots et ce qu'ils désignent à partir du concept d'hétérogénéité pour en souligner la dimension langagière créative en analyse de l'activité. Dans le dialogue réalisé entre le professionnel et le chercheur-psychologue, on étudie alors la fonction occupée par ce dernier dans ce processus développemental, notamment les effets produits par sa reprise des mots de l'autre. Ce qui apparaît comme problématisation de la parole d'autrui devient un instrument majeur pour favoriser le « penser-dire autrement » (François, 2001, p. 108).

En fin d'article on tente de définir en quoi les points travaillés dans ce texte contribuent à caractériser le processus de bi-vocalisation. Ce processus, lorsqu'il se déploie, signe le développement des discours et des manières de penser l'activité en organisant la rencontre avec la parole d'autrui sur l'objet même.

\section{1.- Le dialogisme $e^{2}$ bakhtinien : une conception étendue du dialogue}

C'est jusqu'à la fin de son activité d'écriture que Bakhtine approfondira la théorie du dialogisme à la lumière notamment de l'œuvre de Dostö̈evski.

\section{1.- Une conception historique et sociale du langage : l'énoncé bakhtinien}

L'énoncé bakhtinien, « unité réelle de l'échange verbal », est de nature sociale et historique.

Ses principales caractéristiques se décrivent selon trois points fondamentaux : le caractère achevé de l'énoncé, sa triple orientation vers l'objet, vers les autres mais aussi vers soi et enfin sa dimension dialogique: parler à autrui, c'est transformer des énoncés existants tout autant qu'anticiper la réaction de son interlocuteur.

Bakhtine décrit « l'achèvement spécifique de l'énoncé » comme étant un indice fondamental de celui-ci. Les énoncés possèdent toujours « des frontières nettement délimitées » par l'alternance des sujets parlants (1984, p. 277). Ils se terminent par « le transfert de la parole à autrui » et deviennent, dans le dialogue vivant, des « répliques »: « chaque réplique, aussi brève et fragmentaire soit-elle, possède un achèvement spécifique qui exprime la position du locuteur - il est possible de répondre,

social entre la signification et le monde (Clot, 1999, p. 173). Il attire ainsi notre attention sur l'absence de rapports directs entre le sujet, la langue et le monde.

2. Il ne s'agirait pas de réduire le terme de dialogisme à celui de polyphonie, emprunté au domaine musical, et qui désigne la pluralité des voix. Dans ses écrits, Bakhtine utilise le mot polyphonie uniquement lorsqu'il étudie la structure du roman dans l'œuvre de Dostoïevski pour décrire la singularité du texte. Les traductions françaises, comme le souligne Nowakowska (2005), ont étendu exagérément l'usage du mot polyphonie au point de le vider de sa signification propre ou pour le moins de gommer toutes les distinctions avec le dialogisme. Selon elle, « si l'on veut rester fidèle à la lettre du texte de Bakhtine », il est judicieux « de réserver le terme de polyphonie au domaine littéraire, et plus précisément encore à un certain type de roman; et de ne parler, pour la parole quotidienne, que de dialogisme » (p. 26). La distinction majeure entre les deux notions se situe sur le rapport entre les différentes voix: le dialogisme contrairement à la polyphonie souligne la prépondérance d'une voix sur les autres, il relève la hiérarchisation des voix. Mais la pluralité des voix intègre aussi celles du dialogue interne, « le monologue intérieur de chacun de nous ressemble à une sorte de théâtre où l'on ne sait plus très bien qui parle, une sorte de polylogue anonyme » (François, 2005. p. 22) et l'existence d'un passage, d'une « suture » pour reprendre l'expression de Peytard (1995, p. 95) entre l'interne et l'externe. 
il est possible de prendre, par rapport à cette réplique, une position responsive » (id., p. 278). À partir d'une certaine représentation du « vouloir-dire » de son interlocuteur, il devient possible d'enchaîner une réplique à l'énoncé précédemment produit.

L'énoncé, dans la conception bakhtinienne du dialogue, ne se réduit pas à ce premier aspect puisqu'il se définit également comme une triade vivante tournée vers l'objet, vers autrui et vers soi. Bakhtine écrit dans Esthétique de la création verbale: « très souvent l'expression de notre énoncé est déterminée non seulement (...) par la teneur de l'objet de notre énoncé, mais aussi par les énoncés d'autrui sur le même thème auxquels nous répondons, avec lesquels nous polémiquons. (...) L'expression d'un énoncé (...) manifeste non seulement son propre rapport à l'objet de l'énoncé, mais aussi le rapport du locuteur aux énoncés d'autrui » (1984, p. 299). Ce rapport est dialogique puisque parler à autrui c'est tout autant transformer des énoncés existants que se situer dans la perspective de la réponse à venir. La dimension en effet sur laquelle Bakhtine et Volochinov insistent dans leur théorie de l'énoncé est celle de l'historicité: "Il est donc impossible de comprendre comment se construit un énoncé quelconque, eut-il l'apparence de l'autonomie et de l'achèvement, si on ne l'envisage pas comme un moment, comme une simple goutte dans ce fleuve de la communication verbale dont l'incessant mouvement est celui-là même de la vie sociale et de l'histoire » (Volochinov, 1930, trad. Todorov, 1981, p. 288). Ni les mots, ni les « choses » ne sont neufs, ils portent la trace d'autres discours, ce qui fait dire à Bakhtine que le locuteur lui-même est en quelque sorte « un répondant ». Parler à autrui, c'est aussi anticiper sa réponse et pour Bakhtine, le locuteur seul n'existe pas. Celui qui parle (ou qui écrit) oriente en permanence ses propos en direction d'autrui, et plus particulièrement en direction de la réponse qu'il anticipe de sa part. L'auditeur revêt une autre posture que celle de récepteur, y compris lorsque l'on attribue à ce dernier l'identité de « sujet-destinataire ». Ainsi que Grossen le résume, à partir de Bakhtine, «the interlocutor's possible response is contained within the speaker's discourse and any piece of discourse has one component made up of responsivity and another of addressivity » (à paraître).

L'énoncé dans la théorie du dialogisme est donc doublement imbriqué dans la prise en compte d'une part des énoncés déjà-là - tous les autres discours du milieu et non pas une langue comme système abstrait - et d'autre part dans l'anticipation des énoncés-réponses.

Cette conception de l'énoncé invite à prendre en compte « les circuits interdiscursifs » (Schepens, 1999, p. 37) dans l'étude du dialogue et s'oppose à une vision restreinte du dialogue en tant qu'échange entre deux interlocuteurs.

\section{2- Le concept d'hétérogénéité}

Le principe dialogique de Bakhtine pose, comme nous venons de le souligner, que tout discours se fait dans son rapport à d'autres discours: tout autant son rapport à l'ensemble des autres discours existants dans le «milieu » desquels il s'élabore - en référence au « genre »- mais aussi plus spécifiquement son rapport à cet autre discours de celui à qui il s'adresse. Le discours est ainsi tissé des mots d'autrui et dès l'entrée dans le langage, les mots sont des mots des autres. On ne rencontre que des «mots occupés » pour reprendre la formule de Bakhtine : « aucun mot n'est neuf, ni neutre, mais « chargé » d'un « déjà-dit », celui des contextes où il a « vécu sa vie de mot »; en ce sens «parler c'est entrer en rapport dialogique avec ces discours autres qui habitent les mots et c'est dans le processus - qui écoute, accueille, avec ou sans réticence, chaque mot en fonction de ce qui résonne en lui de voix étrangères - que le discours «prend corps » (Authier-Revuz, 2000, p. 37). "L'hétérogénéité » c'est-à-dire la présence d'un discours autre dans le discours de l'un recouvre ainsi selon Authier-Revuz deux dimensions : elle est à la fois « constitutive » mais aussi «montrée » lorsque la présence localisable d'un discours autre dans le fil du discours est observable.

Pour cette auteure, toute énonciation se produit, c'est-à-dire « prend corps » en négociant avec l'altérité selon quatre modalités:

- Dans la non-coïncidence entre les interlocuteurs irréductiblement singuliers l'un par rapport à 
l'autre (je te parle de..., comme vous dites...);

- Dans la non-coïncidence du discours avec lui-même, les discours étant traversés par l'ailleurs du déjà-dit d'autres discours comme en témoigne le discours rapporté sous forme directe ou indirecte (comme dit untel...);

- Dans la non-coïncidence entre les mots et les choses, entre la langue et l'expérience du réel inscrite dans la singularité: les mots et la chose ne font pas un, il n'y a pas identité de nature entre l'un et l'autre (comment dire? Ou c'est le mot qui convient ou encore, je ne sais pas si c'est le bon mot). La non-coïncidence entre le mot et la chose qu'il désigne se montre nettement lorsqu'on observe le caractère réflexif du langage c'est-à-dire lorsque le sujet parle de quelque chose et simultanément, « il se dit disant » (Salazar-Orvig, 1999, p. 24);

- Dans la non-coïncidence des mots à eux-mêmes, la non-univocité des signes [plusieurs sens possibles pour le même mot (au sens figuré, à tous les sens du mot...)].

De cette dépendance aux mots des autres et dans cette recherche du mot juste, le locuteur parvient avec plus ou moins de facilités, plus ou moins de réussite, de bonheur à élaborer des mots à soi. Les mots autres deviennent habités par le sujet, il les charge d'une signification en propre dans un processus situé à l'intersection de la vie intérieure et extérieure.

La question que nous regardons ici part de ce point précis du processus pour le suivre: comment un mot ainsi chargé d'une signification en propre se développe-t-il? C'est Bakhtine lui-même qui autorise à placer cette réflexion sous le sceau du dialogisme: pour lui le rapport dialogique, possible entre deux énoncés de voix différentes, peut aussi se réaliser vis-à-vis de notre propre énoncé, « si nous prenons du recul par rapport à cet énoncé, si nous ouvrons des parenthèses intérieures, s'il y a une distanciation par rapport à lui » (1970, p. 256).

Mais avant de nous intéresser aux processus dynamiques et aux conditions dialogiques de ces mouvements langagiers créatifs entre objectivation et subjectivation, il va s'agir tout d'abord de préciser les modalités de production des données que nous étudions.

\section{2.- Méthodes d'analyses de l'activité et activité analysée}

En analyse du travail, les méthodes associent régulièrement les acteurs de la situation à son étude. Pour ce qui concerne la méthode des autoconfrontations croisées (Clot et al., 2001), qui a été ici mobilisée, il s'agit pour le psychologue de mettre en place et seconder une activité réflexive des professionnels à partir de films d'activité. Délimitée avec le collectif professionnel associé à la recherche, à partir d'une commande et/ou d'une demande et d'un long travail de co-conception, une séquence de l'activité est d'abord filmée. Chacun des protagonistes la commente ensuite individuellement avec le chercheur à partir du film réalisé - autoconfrontation simple - après quoi chacun commente l'activité de son collègue ayant réalisé la même tâche que lui, avec celui-ci - autoconfrontation croisée - toujours à partir des films et toujours en présence du chercheur. Ces commentaires de chacun sur son travail et sur celui de l'autre sont filmés et sont ensuite présentés au collectif qui peut ainsi reprendre et poursuivre les dialogues engagés. C'est qu'en effet au-delà de la rigueur méthodique, la visée méthodologique est précise: il s'agit de mobiliser les films d'activité et les commentaires à leur sujet comme instrument de l'interprétation par les professionnels eux-mêmes de ce qu'ils font, mais aussi de ce qu'ils auraient pu faire, ce qu'ils n'ont pas pu faire, ce qu'ils pourraient faire d'autre... afin que les collectifs de travail eux-mêmes puissent revitaliser ou conserver vivantes leurs ressources d'invention.

C'est ici une activité bien particulière - s'il en est - la Validation des Acquis de l'Expérience (VAE) - qui fait l'objet du commentaire. Institué par un décret datant de 2002, le dispositif permet à des personnes ayant développé une expérience professionnelle de solliciter l'attribution d'un diplôme sur la base de celle-ci. Cette nouvelle voie concerne de multiples institutions, dont notamment tous les ministères qui ont autorité à certifier. Ils doivent alors mettre en œuvre l'ensemble des dispositions 
qui permettent « de vérifier si les acquis (de l'expérience) dont le candidat fait état correspondent aux compétences, aptitudes et connaissances exigées par le règlement de chaque diplôme » (Décret d'avril 2002). Concrètement, ils ont à organiser l'accueil, l'accompagnement des candidats dans leur démarche, la mise en place de jurys, etc.

Préoccupé par les nouvelles questions que pose ce dispositif, c'est l'un de ces ministères ${ }^{3}$ qui sollicite une intervention des chercheurs. Précisément, il s'agit pour ses responsables de faire de la VAE un moyen de certification aussi fiable que les autres ${ }^{4}$. Pour ce faire, on y postule que l'analyse du travail peut être un moyen de développer l'organisation et les actions conduites en VAE. Plus précisément, celles qui apparaissent initialement comme les plus problématiques: la lecture du dossier «d'expériences » du candidat à la VAE et l'entretien avec celui-ci, activités chargées l'une et l'autre de l'enjeu important attaché à la décision de délivrer ou non un diplôme. Les professionnels qui prennent en charge la VAE n'ont pas été nouvellement recrutés à cette fin. Ils sont ou ont été animateurs, formateurs, ingénieurs de formation au sein de structures régionales ou départementales et la VAE vient s'ajouter à d'autres activités plus classiques.

Certains d'entre eux se sont portés volontaires pour conduire l'analyse précise de leur activité de lecture de dossier ou d'entretien.

Nous n'entrerons pas dans les détails de l'ensemble des activités complexes du processus de validation. Mais il nous faut cependant préciser les éléments relatifs à l'entretien avec le candidat puisque c'est lui qui constitue l'objet du dialogue que nous proposons d'analyser. L'entretien avec le candidat suit la lecture du dossier par les membres du jury et doit permettre « de compléter les points du dossier dont la formulation ne serait pas jugée assez précise pour traduire la mise en œuvre des compétences visées $\gg .5$

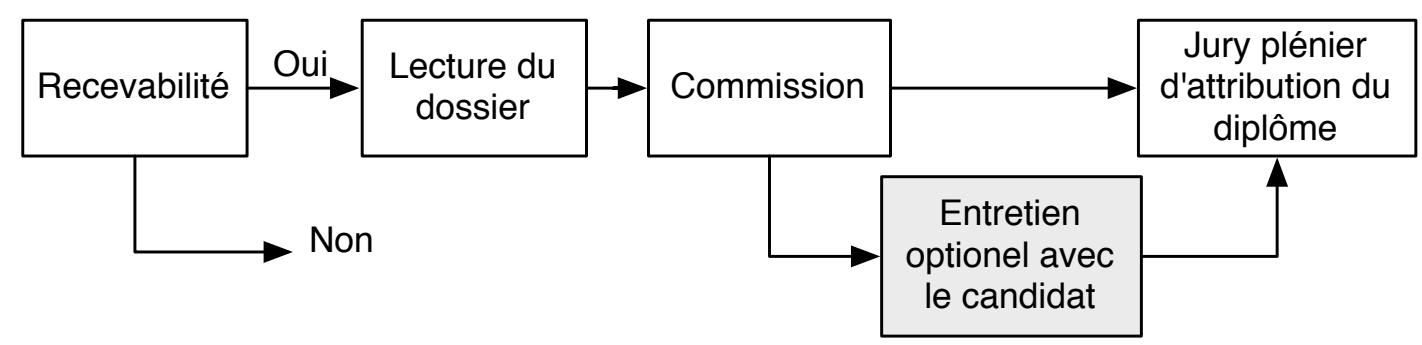

Figure 1.- Le processus de validation des acquis de l'expérience

Validation of experience work's process

Ordonné par une prescription très précise, l'entretien, optionnel, est initié par la commission ou s'effectue à la demande du candidat. D'une durée maximale de $30 \mathrm{mn}$, réalisé par les membres de la commission de lecture du dossier, il doit porter sur le dossier écrit et ne peut être un « oral de rattrapage », non plus qu' « une soutenance de rapport de stage » ou un « exposé ». Les questions doivent être «préparées » au sein de la commission de lecture et « réparties » entre ses membres, qui doivent « conduire » l'entretien après en avoir « présenté le cadre ». Des exemples de questions à poser ou à éviter complètent le texte prescriptif. De nombreuses difficultés trament pour les professionnels la réalisation de cette activité : comment conduire l'entretien, installer le cadre, etc. Dans la situation étudiée, ce qui fait l'objet du commentaire, ce sont les premiers mots que les deux membres de la commission mandatés pour mener cet entretien prononcent lorsque le candidat arrive et s'installe. Ils sont préoccupés par la nécessité de devoir recueillir des informations suffisantes et importantes

3. A l'époque Ministère de la Jeunesse, des Sports et de la Vie Associative.

4. Les moyens qui sont attachés au suivi d'une formation et à la passation de ses épreuves, en formation initiale, continue ou en apprentissage.

5. Ces compétences sont définies dans le document suivant: Instruction nº2-183 du 6 novembre 2002 relative à la validation des acquis de l'expérience. Ministère de la jeunesse et des sports. 
en un temps limité.

\section{3.- Analyse des extraits de dialogues en autoconfrontation simple}

L'activité d'entretien avec le candidat, filmée, a duré $22 \mathrm{mn}$ et l'autoconfrontation simple a été réalisée à la suite de cet enregistrement. Elle dure au total une heure trente.

Nous en avons retenu deux séquences qui prennent place dans les premières quarante répliques du commentaire, qui en comporte environ cent cinquante. Ces passages ont été sélectionnés pour des motifs bien précis : les répliques condensent de nombreuses traces d'hétérogénéité et formellement, un même mouvement semble s'y répéter. On observe en effet une rupture manifeste dans le fil du dialogue lorsque le chercheur s'adonne à la reprise des mots de son interlocuteur. Le phénomène est d'autant plus frappant que l'autoconfrontation simple conduite avec un autre professionnel sur la même tâche avec le même intervenant ne présente aucune de ces spécificités.

Le commentaire s'est porté là dès la première minute, sur la manière d'accueillir la candidate, avec l'avènement d'une règle qui définit l'orientation de l'entretien: mettre le candidat à l'aise.

\begin{tabular}{|c|c|c|}
\hline $\mathrm{Ch}^{\mathrm{a}}$ & 7 & c'est important que les candidats soient à l'aise pour .../ \\
\hline $\mathrm{JF}^{\mathrm{b}}$ & 8 & / oui oui \\
\hline $\mathrm{Ch}$ & 9 & pour la suite? \\
\hline $\mathrm{JF}$ & 10 & $\begin{array}{l}\text { je pense oui c'est important... en tout cas dans toutes les conditions que ce soit VAE } \\
\text { ou une autre condition d'examen ...des oraux // on essaie de mettre ... le candidat à } \\
\text { l'aise // et aussi chose qu'on fait toujours c'est se présenter // dire... à quel titre ... on } \\
\text { parle // donc... donc....quand on est membre du jury //c'est pour les candidats c'est } \\
\text { important de savoir... aussi... quel est le statut quoi la position dans le jury... si c'est } \\
\ldots \text {..je dirais un représentant des syndicats employeurs des syndicats employés là c'était } \\
\text { une une... formatrice mais ça aurait pu aussi // les candidats je pense quelque part } \\
\text { derrière ça // ils ont... ça les ...ça peut aussi les guider (sourire) ça les aide et puis } \\
\text { c'est pas c'est pas un entretien anonyme (inaudible) }\end{array}$ \\
\hline $\mathrm{Ch}$ & 11 & quand vous dites que ça peut les guider? \\
\hline $\mathrm{JF}$ & 12 & $\begin{array}{l}\text { alors je sais pas si c'est guider mais enfin c'est nous c'est // bon } / / \text { je sais pas j'ai pas } \\
\text { trop analysé } / /(2 s) \text { en tout cas on a l'habitude de le faire (rire) c'est pour ça et ...on } \\
\text { se pose rarement la question de savoir } / /(2 s) \text { non c'est d'où t'on d'où on parle je crois } \\
\text { je veux dire on est aussi ....c'est un acte qu'on fait en pédagogie aussi on s'exprime } \\
\text { toujours ... de ... de quelque... enfin on dit toujours de ... // qui on est quand on ... } \\
\text { donc on n'est pas dans une attitude vraiment pédagogique puisque là on est dans la } \\
\text { certification on n'est pas en formation mais// ou dans l'évaluation mais... je pense ça } \\
\text { a une importance pour les personnes de savoir ...ben qui on est aussi ça fait partie des } \\
/ / \text { de ce qu'on peut enseigner aussi (rire) le savoir être a tellement d'importance dans } \\
\text { nos... dans nos métiers et dans les métiers qu'ils... que font aussi les candidats les } \\
\text { animateurs que c'est important de savoir qui ils ont en face }\end{array}$ \\
\hline
\end{tabular}

Tableau 1: Extrait d'autoconfrontation simple ${ }^{\circ} 1$

(a: Chercheur, b: Initiales du professionnel)

Table 1 : Self confrontation's extract 1

Dans la seconde séquence, retranscrite ci-dessous, les manières de présenter apparaissent variables: JF ne travaille pas toujours de la même manière, notamment selon que l'entretien est à l'initiative de la commission ou à celle du candidat. Il découvre par ailleurs qu'il ne fait pas ce qu'il pensait faire: demander à la candidate de se présenter. La retranscription présentée fait d'abord référence à la situation vue à l'écran (en gris), celle de l'entretien avec la candidate, puis au commentaire de JF sur ces mêmes images. 
JF s'adresse à la candidate

// (3sec) donc ....alors c'est document très riche hein donc on... bien sûr on va pas tout retracer//

hein là est bien sur une demande un peu plus d'explications sur certains points qui nous a semblé ...demander p... un peu plus d'informations // ... je rappelle quand même que votre dossier a été vu par quatre personnes c'est-à-dire que nous sommes là délégués un peu en quelque sorte par une commission ... qui a...... de différentes personnes qui ont lu votre dossier sachant que la commission était... composée était plus importante et que tout ça était soumis à au moins .. au regard de huit personnes au final voilà// voilà (grand sourire) // donc ....on voudrait que

\begin{tabular}{|c|c|c|}
\hline \multicolumn{3}{|c|}{ Commentaire en autoconfrontation simple } \\
\hline $\mathrm{Ch}$ & 33 & $\begin{array}{l}\text { vous reprécisez que vous n'êtes pas les deux seules personnes impliquées c'est } \\
\text {....important? }\end{array}$ \\
\hline $\mathrm{JF}$ & 34 & $\begin{array}{l}\text { oui pour le candidat je pense que // ... ça c'est .....c'est c'est... c'est... pour ... lui } \\
\text { montrer qu'il y a eu des regards croisés quoi je veux dire sur son // sur son dossier } \\
\text { qu'il a été lu par... attentivement par des personnes différentes et que..... bon c'est } \\
\text { pas...... c'est c'est c'// que ça fait l'objet de discussions bon on le dit pas mais } \\
\text { j'espère que... c'est implicite pour tout le monde mais enfin... on espère j' espère que } \\
\text { c'est implicite vi... implicite aussi pour la candidate que son dossier a été....// rappeler } \\
\text { quoi je veux dire l'intérêt qu'on a pu porter// voilà je veux dire je crois que c'est } \\
\text { important ... quand on voit ben l'épaisseur... bon le travail qu'elle a pu réaliser// sur } \\
\text { ce dossier là moi je crois que c'est c'est déjà bon c'est une forme de reconnaissance } \\
\text { quoi je veux dire en disant ben le dossier n'a pas été lu par une personne sur un coin } \\
\text { de table et puis voilà on a préparé un questionnement... rapidement non// y'a.... une } \\
\text { commission et cette commission en plus... a soumis ça... a soumis son avis à une } \\
\text { autre... parce qu'il y a deux commissions en parallèle ...parce qu'on avait beaucoup } \\
\text { de... dossiers donc... et donc... pour aussi.... des questions d'équité entre tous les } \\
\text { candidats/.... }\end{array}$ \\
\hline $\mathrm{Ch}$ & 35 & $\begin{array}{l}\text { et le dire à la candidate vous disiez c'est une forme de reconnaissance par rapport à } \\
\text { elle? }\end{array}$ \\
\hline JF & 36 & $\begin{array}{l}\text { ben oui du... je veux dire du travail qu'elle a (amorce de rire) pu accomp accomplir } \\
\text { quoi alors reconnaissance.... je sais pas si c'est le mot mais enfin en tout cas // dire } \\
\text { que... son dossier a été... lu avec intérêt (rire) et que... on y a porté cas et que... } \\
\text { c'est c'est important qu'il y ait plusieurs lecteurs quoi // (2 sec) je sais p// c'est } \\
\text { aussi pour...// (2sec) comment je vais dire... éviter.. oui que... que... la subjectivité } \\
\text { quoi je veux pas dire qu'on est objectif (rire) mais on essaie d'avoir d'aller vers le } \\
\text { plus d'objectivité possible donc// en disant que le... le nombre de lecteurs est plus } \\
\text { important c'est...// je pense//... pour...// pour le candidat ça doit le ...le... comment } \\
\text { je vais dire... ça doit être plus im c'est important pour qu'il comprenne que c'est plus } \\
\text { objectif...// c'est pas sûr hein ... (rire) là j'ai aucun moyen de vérifier si elle a bien } \\
\text { compris ça mais enfin bon c'est... c'est dit dans cet inter... dans ce cadre là }\end{array}$ \\
\hline
\end{tabular}

Tableau 2: Extrait d'autoconfrontation simple $\mathrm{n}^{\circ} 2$

Table 2: Self confrontation's extract 2

L'analyse suivra ici un enchaînement précis permettant de suivre pas à pas la trace des mouvements discursifs dans l'activité dialogique: on distinguera tout d'abord la possibilité d'un dialogue avec soi-même - dans ses caractéristiques de distanciation -, puis le dialogue avec l'interdiscours dans le discours en cours de réalisation et enfin le dialogue entre chercheur et professionnel - en particulier les effets de la reprise des mots de l'autre. Le dialogue est, ne l'oublions pas, «plus étendu, plus varié, plus complexe » (Bakhtine, 1984, p. 334) que le dialogue réel. Mais le dialogue « réel » constitue cependant la condition du développement des deux autres.

Le constat intuitif de départ dans l'analyse des données s'appuyait sur les nombreuses ruptures, les 
silences, autrement dit l'hétérogénéité qui marquait la réalisation de ce commentaire. En référence aux travaux d'Authier-Revuz, on peut avancer que la caractéristique de ces traces relève de façon majoritaire de ce que cette auteure définit comme les formes de non-coïncidence entre les mots et les choses. On rappelle que celles-ci se manifestent lorsque l'énonciateur représente son acte propre de nomination dans sa certitude, ses hésitations, son échec, sa surprise... C'est tout particulièrement au cours des répliques JF 12 (extrait $n^{\circ} 1$ ) et JF 36 (extrait $n^{\circ} 2$ ) que se condense ce jeu de distanciation, par ailleurs répétée dans tout le dialogue. JF exprime nettement ce rapport non direct entre les mots et les choses:

- En émettant explicitement des doutes sur la nomination (« je ne sais pas si c'est guider... je ne sais pas... », « je ne sais pas si c'est le mot... »),

— En manifestant son désir de dire («...je veux dire...»),

— En rendant visible ses difficultés de nomination («...comment je vais dire...»),

— En étant partagé dans le choix des mots («... je veux pas dire...»).

Toutes ces formes « dénaturalisent l'évidence de l'association mot-chose, pour inscrire l'image d'un dire comme travail de production -élaboration nomination, comme trajet dans le champ des possibles » (Authier-Revuz, 1995, p. 606). La progression dans la nomination est sujette à une monstration peu commune: elle offre de nombreuses occasions de déploiement de la pensée dans les mots.

Mais si la caractérisation constitue un premier moment de l'analyse, ce sont « les mouvements discursifs, les affinités, les récurrences et les ruptures » (Salazar-Orvig, 1999, p. 9) qui peuvent rendre compte du développement en cours.

Il apparaît ici une première récurrence dans le discours de JF à savoir la mobilisation de mots qui définissent ce qu'il fait «d'habitude», dans un entretien. Par exemple, «on leur dit de s'asseoir puis de se mettre à l'aise » (JF6, extrait $\left.\mathrm{n}^{\circ} 1\right)$ ou encore "c'est un acte qu'on fait en pédagogie aussi on $s^{\prime}$ exprime toujours » $\left(\mathrm{JF} 12\right.$, extrait $\left.\mathrm{n}^{\circ} 1\right)$ : le « on » associé au présent signe le discours générique. La mémoire des manières de dire et d'agir est mobilisée pour se débrouiller dans la situation en cours. Les mots ou les formules sont chargés d'un ailleurs mais aussi d'une certaine façon prisonniers des contextes dans lesquels ils ont circulé et ce lestage, ce préconstruit s'invite dans l'interlocution. Si cette «mémoire discursive » comme la nomme Sitri (2003, p. 101), en prise directe avec la mémoire du métier habituellement exercé, ponctue à intervalles réguliers le discours, d'autres réalisations émergent simultanément. Relevons par exemple: "on n'est pas dans une attitude vraiment pédagogique puisque là on est dans la certification on n'est pas en formation » (JF 12, extrait $\left.\mathrm{n}^{\circ} 1\right)$ ou encore « reconnaissance je sais pas si c'est le mot/../c'est pour montrer qu'on est plus objectif » (JF 36, extrait $\mathrm{n}^{\circ} 2$ ). Il y a ainsi mise en concurrence constante dans le discours de JF entre ce qu'il fait habituellement et ce qu'il se voit faire en visionnant le film de son activité, les mots devenant les arènes où se réalisent ces mises en tension. Au bout du compte l'impossibilité de se cantonner à dire du déjà-dit contraint à l'élaboration d'autres modalités du «penser-dire ». Cet «ébranlement »du discours prépare la voie à la discussion collective, elle en constitue même un jalon essentiel.

Attachons-nous à présent à étudier la reprise comme processus de problématisation de la parole d'autrui, seconde récurrence marquant la dynamique interlocutive. Dans les extraits présentés mais aussi dans l'ensemble du dialogue, la plupart des interventions du chercheur se présentent sur ce mode de la reprise ${ }^{6}$ des mots de JF. Celle-ci s'organise selon deux modalités. Dans un premier cas, la reprise porte sur un mot utilisé par JF dans le commentaire en cours, avec un passage de la forme affirmative à la forme interrogative « quand vous dites que ça peut les guider? » (Ch 11, extrait $\left.n^{\circ} 1\right)$; « et le dire à la candidate, vous disiez c'est une forme de reconnaissance par rapport à elle? »(Ch 35 , extrait ${ }^{\circ} 2$ ). Dans un second cas, la reprise porte sur des énoncés de JF dans le cadre de l'entretien avec la candidate, à partir du film visionné. Elle mobilise une formulation différente de celle

6. Nous choisissons d'utiliser le terme de « reprise » proposé notamment par Vion (2006), de préférence à « reformulation » : sa caractéristique générique permet de considérer une relation au déjà-dit, dont les rapports et les formes de similitude peuvent varier. 
employée au cours de l'énonciation initiale. JF a, par exemple, évoqué un dossier « vu par quatre personnes » puis «le regard de 8 personnes » (entretien avec la candidate, extrait $\mathrm{n}^{\circ} 2$ ). La reprise par le chercheur transforme ainsi les énoncés: «vous n'êtes pas les deux seules personnes impliquées?» et sollicite une évaluation «c'est important ? » (Ch 33, extrait n²).

Ces deux modes de reprise sont importants à distinguer car ils génèrent des répliques différentes de la part de JF. Nous y reviendrons. Mais on peut dès à présent remarquer que ces reprises, telles qu'elles s'observent dans ces répliques, possèdent de façon nette des vertus explicitantes, le professionnel étant entraîné dans une réévaluation de son activité. Elles provoquent des développements dont le mode de progression est paradoxal. On y constate en effet un double mouvement en simultané de continuité et de rupture:

- La continuité s'observe dans le recours à du déjà fait: «...on a l'habitude de le faire... c'est un acte qu'on fait en pédagogie, on s'exprime toujours... » (JF 12, extrait $\left.\mathrm{n}^{\circ} 1\right)$ ou à du déjàdit « ... c'est important qu'il y ait plusieurs lecteurs » (JF 36), paraphrase de l'énoncé JF 34 « ....sur son dossier qu'il a été lu par... attentivement par des personnes différentes » (extrait $\left.\mathrm{n}^{\circ} 2\right)$.

- La rupture quant à elle s'observe d'une part dans le décrochage méta-énonciatif, « alors je sais pas si c'est guider... » (JF12, extrait $\left.\mathrm{n}^{\circ} 1\right)$, ou encore « je ne sais pas si c'est le mot » $(\mathrm{JF}$ 36 , extrait $n^{\circ} 2$ ) rompant l'évidence du rapport mot-chose et d'autre part dans les nouveaux développements, «c'est aussi pour... » (JF 36, extrait n ${ }^{\circ}$ ).

Un effet de sens se produit dans la répétition des mots de l'autre du fait de la mise en valeur d'un mot ou d'une formule par rapport à l'ensemble de l'énoncé.

Mais l'on peut alors revenir à la différence entre les deux modes de reprise ci-dessus identifiées. En effet, plus la reprise est indirecte (Ch7, Ch33), et plus l'identité verbale du locuteur premier s'efface, moins celui-ci assume en quelque sorte la responsabilité de ses propos. À l'inverse plus les mots que le locuteur entend sont identiques à ses propres mots $(\mathrm{Ch} 11, \mathrm{Ch} 35)$ et plus la prise en charge énonciative de ceux-ci devient importante. La signification attribuée au mot recouvre un enjeu important pour le locuteur et devient le centre d'un dur « combat dialogique » pour reprendre la formule de Bakhtine.

Si la reprise est un «principe fondamental de l'activité discursive » (Salazar-Orvig, 2000, p. 68), c'est en tant qu'elle présente les propriétés «d'un énoncé dialogique » puisqu'à travers cette reprise « se joue une véritable confrontation entre deux énonciations, telle que la conçoit Bakhtine » (Bernicot, Salazar-Orvig, \& Veneziano, 2006, p. 34). Une deuxième occurrence, même formellement identique à la première, "représente un fragment nouveau d'énoncé car sa position et sa fonction ont changé dans le tout de l'énoncé » (Bakhtine, 1984, p. 317). C'est bien la caractéristique de la reprise relativement à d'autres formes de dialogisme: l'hétérogénéité est paradoxalement marquée par une relation de similitude formelle. Dans ce mouvement, un décalage devient possible entre la signification attribuée en premier par l'énonciateur et toutes les autres significations possibles que le mot ou la formule peuvent contenir. Bakhtine écrit à ce sujet dans La poétique de Dostoïevski que « le simple fait de reproduire l'affirmation d'autrui sous forme de question amène l'affrontement entre deux interprétations dans un même mot, nous ne nous contentons pas d'interroger, nous problématisons l'affirmation d'autrui » (p. 269). C'est que pour lui le mot ne se réduit pas à sa seule signification, l'intonation fait partie de sa vie concrète: le même mot, avec une autre intonation, est un autre énoncé et devient inachevé.

À ce point, demeure une caractéristique soulignée mais non encore prise en compte dans l'analyse: celle du mouvement interlocutif qui oscille entre d'une part la reprise des mots prononcés par JF dans l'entretien avec la candidate et d'autre part la reprise des mots prononcés dans le commentaire en cours. C'est dans ce dernier cas, qui coïncide avec les répétitions les plus directes, que ruptures et développements sont les plus manifestes relativement au déjà-dit ou au déjà fait. Mais c'est dans cette situation aussi que les développements qui s'ensuivent font coexister deux manières de dire et penser 
l'action. C'est en particulier net en JF 36 (extrait $\mathrm{n}^{\circ} 2$ ), réplique au cours de laquelle reconnaître, porter intérêt peut se lier avec être objectif. Or, les deux registres, selon notre connaissance de la situation et plus généralement une connaissance de la VAE, sont très différents : l'un concerne la prise en compte du candidat, l'autre est tourné vers l'activité d'évaluation et le jury. On avancera alors que l'interlocution dans ses mouvements contribue à réaliser les dilemmes entre l'activité antérieure, fortement ancrée dans une histoire professionnelle de la pédagogie, et l'activité nouvelle d'entretien de jury délivrant un diplôme à partir de l'expérience. La dénaturalisation des mots habituels pour dire et penser l'action, la déliaison entre le mot et la chose pour l'écrire à la manière d'Authier-Revuz, que la répétition du mot précipite, se développe en une autre liaison avec l'action: sans oublier l'histoire (reconnaître), elle la renouvelle à cette occasion (être objectif).

La reprise dans le fil du dialogue en autoconfrontation simple est mobilisée quand le chercheur ne comprend pas ou n'est pas certain d'avoir compris la signification attachée aux mots. Elle est aussi convoquée lorsque le professionnel lui-même manifeste de l'étonnement par rapport à ce qu'il se voit faire ou s'entend dire. Mais la mobilisation de la reprise dans le fil du dialogue n'est pas suivie systématiquement d'un effet de problématisation de la parole par celui qui s'entend répéter ses propres mots. D'autres voies sont parfois empruntées. C'est le cas dans les extraits ci-après, issus d'une situation comparable: la même tâche, sur la même activité, celle de l'entretien avec le candidat en VAE, avec le même intervenant. Un autre professionnel en revanche commente alors le film de son activité:

\begin{tabular}{|c|c|c|}
\hline Pro & 24 & //.../ cette affaire là \\
\hline $\mathrm{Ch}$ & 25 & cette affaire là c'est-à-dire? \\
\hline Pro & 26 & le contrat qu'on passe avec eux et comment va se passer l'entretien /.../ \\
\hline \multicolumn{3}{|r|}{ I.../ } \\
\hline Pro & 43 & /.../ la voit pas $/ . . . /$ \\
\hline $\mathrm{Ch}$ & 44 & vous dites on la voit pas? \\
\hline Pro & 45 & Lnon on la voit pas donc je cherche $/ . . . /$ \\
\hline \multicolumn{3}{|r|}{ I.../ } \\
\hline Pro & 117 & /.../ là je reprends la main/.../ \\
\hline $\mathrm{Ch}$ & 118 & vous dites je reprends la main? \\
\hline Pro & 119 & oui je reprends la main parce que j'avais d'autres questions et je me suis dit... \\
\hline
\end{tabular}

Tableau 3: Extrait d'autoconfrontation simple $n^{\circ} 3$

Table 3 : Self confrontation's extract 3

Les micro-choix opérés dans le cours de l'activité s'énoncent sur un mode assertif, ils ne sont pas placés au carrefour de différents possibles.

\section{4.- Organiser les conditions de la rencontre avec les mots d'autrui}

Les analyses ci-dessus proposées visent à identifier dans la vie des mots, les moments où ceux-ci se chargent de nouvelles orientations interprétatives. Celles-ci se produisent dans le développement de la signification, lorsque le mot se remplit d'autres vies possibles à l'intérieur du dialogue, lorsqu'il n'est pas chosifié. C'est dans la rencontre avec les mots d'autrui que le processus d'intersignification est potentiellement contenu. Bakhtine, dans la «poétique de Dostö̈evski » lorsqu'il définit ce qu'est la «bivocalisation », décrit avec précision ce processus. Il expose le principe selon lequel, « deux significations (interprétations) incarnées ne peuvent se côtoyer comme deux choses inertes, elles doivent se toucher intérieurement, c'est-à-dire entrer dans une liaison de signification » (1970, p. 261). 
On a pu montrer, dans d'autres travaux de recherche, que dans les dialogues entre professionnels, le processus n'est pas toujours développemental, notamment si le dialogue quitte l'objet: il dégénère alors en vaine rhétorique (Bournel Bosson, 2005).

En déplaçant le champ à l'analyse de l'autoconfrontation simple entre un professionnel et un chercheur, on montre que le passage du mot monovocal au mot bivocal peut s'engager à partir de la répétition du mot avec une autre accentuation. Alors, comme le dit Bakhtine, dans « un seul mot [se trouvent] deux orientations interprétatives, deux voix » (1970, p. 262), « deux énoncés également et directement orientés vers l'objet» (id., p. 261). Une condition est cependant indispensable dans le déploiement d'un tel processus : il faut en effet « qu'entre l'objet et le discours, s'insère un discours, un accent étranger» (1975, p.148) pour que « l'objet même du mot [soit éclairé] d'une manière inhabituelle, dévoilant des aspects nouveaux » (id., p. 272).

Mais il faut aller plus loin si l'on veut comprendre pourquoi ce passage et les développements que l'on a pu observer ne se produisent pas toujours, ou pas toujours de la même manière. C'est Volochinov qui dès 1929 soutenait l'importance du dialogue « entre le sujet parlant et sa propre parole » (1929/70, p. 212). Il évoquait alors l'une des conditions de son développement en ces termes: un « transfert de l'attention du locuteur » (id., p. 158) de l'objet du discours au discours lui-même, transfert « conditionné par l'attention de l'auditeur » (id.) Le discours prend alors conscience de lui-même « en qualité de discours d'autrui » (id., p. 169).

C'est en cela qu'il est possible de caractériser le « genre communicationnel » (Linell, 1998) de l'autoconfrontation simple et de la situer comme une étape indispensable avant la rencontre avec les mots d'autrui et notamment ceux des pairs. Une mise en dialogue du professionnel avec lui-même est favorisée car il se regarde « à travers les yeux d'autrui ». Le chercheur en voulant faire acte de compréhension devient un élément majeur dans cette recherche de vérité que personne ne détient mais qui circule dans les différents contextes d'analyse de l'activité. Les mots eux-mêmes entrent alors en développement.

On peut revenir ici précisément sur la méthode d'autoconfrontation à travers le parcours de JF. Bien sûr, et il n'est pas vain de le rappeler, les professionnels expérimentés comme JF - ou d'autres - n'attendent pas les chercheurs-intervenants pour développer le « dire-penser autrement » (ibid.). L'intervention, en amont de tout film ou commentaire, crée toutefois une situation dans laquelle chacun devient potentiellement observateur de sa propre activité, sous l'effet de l'observation par un autre. Cette phase est généralement très peu prise en compte dans les analyses du travail. Pourtant, alors, c'est bien d'un dialogue, au sens de Bakhtine, qu'il est question. L'analyse développée ici trouve place dans le temps suivant, lorsque l'autoconfrontation simple offre une nouvelle forme de réalisation, qui se caractérise notamment par l'adresse concrète à un chercheur-intervenant. On observe que ce dialogue entre dans une autre forme: l'autre-interne précédemment installé, ce dialogue entre soi et soi, se redouble d'un destinataire externe, un intervenant qui ne comprend pas et dont l'activité s'oppose à celle du sujet, y compris lorsqu'il répète. C'est le moyen de remettre en question ce qui était opération incorporée, réponse automatique, évidence de l'action et/ou du mot. C'est aussi - et la présente analyse le montre tout autant - le moyen de développer de nouvelles significations et de nouvelles liaisons, entre elles et avec l'objet.

Mais ces développements ne s'arrêtent pas là puisque la phase croisée de la méthode offre l'occasion d'ouvrir un nouveau front du dialogue, celui que l'on conduit avec un pair ayant réalisé la même tâche. Ici, le dialogue s'engagera à partir des différentes manières de conduire un entretien sur une question fondamentale de métier: celle de la « directivité » du dialogue avec un candidat à la VAE (Henry, 2007). Le résultat de l'autoconfrontation croisée en est que chacun dispose pour soi des multiples manières de faire la même chose, ce « clavier » (Clot, Fernandez, \& Scheller, 2007) de ressources possibles. Ici, cette expérience de dialogue est devenue ensuite un instrument pour agir: $\mathrm{JF}$, dans l'action de président de jury VAE mais aussi en tant que formateur, va instaurer le dialogue comme condition du travail bien fait. En particulier, les débats de la commission préparant l'entretien avec le candidat à la VAE vont devenir un point central des démarches. 


\section{Conclusion}

Au départ se trouve un dispositif, la Validation des Acquis de l'Expérience et des acteurs engagés dans la réalisation de cette activité. À celui-ci vient s'adjoindre un second dispositif, d'analyse du travail, qui, à la demande des acteurs eux-mêmes, vise à leur permettre de développer leur action. Conduit par des intervenants-chercheurs, ce dispositif devient l'occasion de produire des connaissances sur le développement de ce genre d'activité. C'est tout particulièrement les conditions de la mise en œuvre du développement qui sont étudiées à un moment précis, celui où est organisée la première confrontation du professionnel à la trace de son activité.

L'analyse proposée dans cet article met en évidence que la reprise des mots du commentaire du professionnel, par le chercheur, provoque un changement de perspective énonciative et suscite un dire autrement. En installant une autre signification potentielle à l'énoncé, qui devient alors une option parmi un ensemble possible, la reprise peut ouvrir ainsi la voie à d'autres manières de penser et d'agir dans la situation de travail.

Les conditions du passage du penser à l'agir nécessiteraient une discussion en tant que telle. Nous préciserons seulement ici qu'il ne s'agit pas - uniquement - là de l'action observable mais du développement des possibilités d'action par rapport aux choses dès lors qu'on les perçoit d'une autre manière. «Comme devant un échiquier, je vois autrement, je joue autrement » (Vygotski, 1934, p. 317).

Selon les situations, d'autres formes que la reprise du mot peuvent être mobilisées en autoconfrontation mais lorsque le processus ici analysé advient, les conditions sont réunies pour que s'engagent les dialogues entre pairs dans le cadre de la phase suivante de la méthode: celle de l'autoconfrontation croisée. La déréification première des significations prépare alors l'espace possible d'un dialogue développemental avec les mots du collègue ayant effectué la même tâche, l'effet attendu n'étant jamais garanti. C'est sur la nature de ces liens entre les processus langagiers créatifs dans l'autoconfrontation simple et ceux de l'autoconfrontation croisée qu'il sera désormais nécessaire de poursuivre les travaux de recherche. Le chantier ainsi ouvert ne sera pas encore à son terme puisqu'il s'agira ensuite d'étudier les modalités de reprise dans le collectif des temps d'analyse du travail précédemment analysés.

\section{BIBLIOGRAPHIE}

Authier-Revuz, J. (1995). Ces mots qui ne vont pas de soi. Boucles réflexives et non-cö̈ncidences du dire. T1, T2. Paris: Larousse.

Authier-Revuz, J. (2000). Deux choses pour une autre; trajets de non-coïncidence, in « Répétition, Altération, Reformulation ». Annales Littéraires de l'Université de Besançon, 701, 37-61.

Bakhtine, M. (1929/1963/1970). Problèmes de la poétique de Dostö̈evski. Lausanne: L'âge d'homme.

Bakhtine, M. (1934/1975/1978). Esthétique et théorie du roman, Paris: Gallimard.

Bakhtine, M. (1952/1979/1984). Esthétique de la création verbale. Paris: Gallimard.

Bernicot, J., Salazar-Orvig, A., \& Veneziano, E. (2006). Les reprises: dialogue, formes, fonctions et ontogénèse. La linguistique, 42/2, 29-50.

Bournel Bosson M. (2005). Les organisateurs du mouvement dialogique: Autoconfrontations croisées et activité des conseillers en bilans de compétences. Thèse de doctorat en psychologie, Paris: CNAM.

Clot, Y. (1999). De Vygotski à Leontiev via Bakhtine. In Y. Clot (Ed.), Avec Vygotski (pp. 165-185). Paris: La Dispute.

Clot, Y. (2005). L'autoconfrontation croisée en analyse du travail : l'apport de la théorie bakhtinienne du dialogue. In L. Fillietaz, \& J.-P. Bronckart (Eds.), L'analyse des actions et des discours en situation de travail. Concepts, méthodes, applications (pp. 37-55). Louvain-la-Neuve: Peeters. 
Clot, Y., \& Faïta, D. (2000). Genres et styles en analyse du travail. Concepts et méthodes. Travailler, 4, 7-42

Clot, Y., Faïta, D., Fernandez, G., \& Scheller, L. (2001). Les entretiens en autoconfrontation croisée : une méthode en clinique de l'activité. Education Permanente, 146, 17-27.

Clot, Y., Fernandez, G., \& Scheller, L. (2007). Le geste de métier: problème de la transmission. Psychologie de l'interaction, 23/24, 109-138.

Ducrot, O. (1984). Esquisse d'une théorie polyphonique de l'énonciation In Le dire et le dit. (pp. 171-233). Paris: Editions de Minuit.

François, F. (2001). La « pensée » dans le langage, sans le langage, à travers le langage, malgré le langage... Ou raconter et penser. In J.-P. Bernié (Ed.), Apprentissage, développement et significations (pp. 93-109). Bordeaux: Presses Universitaires.

François, F. (2005). Interprétation et dialogue chez des enfants et quelques autres. Lyon: ENS.

Grossen, M. (à paraître) Interaction analysis and psychology: A dialogical perspective. In C. Prevignano, \& P. Thilbault (Eds.), Interaction Analysis and Language. Discussing the state of art. Amsterdam: John Benjamins.

Henry, M. (2007). Du travail au langage sur le travail: un développement. Education Permanente, 171, 149160.

Linell, (1998). Approaching Dialogue. Amsterdam: John Benjamins.

Nowakowska, A. (2005). Dialogisme, polyphonie: des textes russes de M. Bakhtine à la linguistique contemporaine. In J. Bres, P.P. Haillet, S. Mellet, H. Nolke, \& L. Rosier. (Eds.), Dialogisme et polyphonie (pp. 19-32). Bruxelles: De Boeck. Duculot.

Peytard, J. (1995). Mickaïl Bakhtine. Dialogisme et analyse du discours. Paris: Bertrand-Lacoste.

Salazar Orvig, A. (1999). Les mouvements du discours. Style, référence et dialogues dans des entretiens cliniques. Paris: L'Harmattan.

Salazar Orvig, A. (2000). La reprise aux sources de la construction discursive. Langages, 140, 68-91.

Schepens, P. (1999). Linguistique dialogique et psychanalyse. Besançon: Presses Universitaires Franccomtoises.

Sitri, F. (2003). L'objet du débat. La construction des objets de discours dans des situations argumentatives orales. Paris: Presses Sorbonne Nouvelle.

Todorov T. (1981). Mikhaïl Bakhtine, Le principe dialogique, suivi de Ecrits du cercle de Bakhtine. Paris: Seuil.

Vion, R. (2006). Reprise et modes d'implication énonciative. La linguistique, 42/2, 11-25.

Volochinov, V.N. (1929/1977). Le marxisme et la philosophie du langage. Paris: les Editions de Minuit.

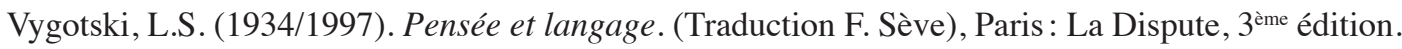

\section{RÉSUMÉ}

Les méthodes d'analyse du travail relevant de la clinique de l'activité engagent les professionnels dans une confrontation aux traces vidéo des actions réalisées. Ce dispositif se veut développemental en tant qu'il ouvre potentiellement des voies pour penser/dire/agir autrement dans le quotidien de l'activité. Dans le cadre de cet article on prend appui sur des données extraites d'une intervention s'inscrivant dans cette orientation méthodologique, conduite avec des acteurs de la Validation des Acquis de l'Expérience. On ouvre une piste dans l'étude du processus langagier créatif en décrivant avec précision l'impact de la reprise des mots du professionnel par le chercheur. Le concept d'hétérogénéité est mobilisé, à partir de Bakhtine, pour comprendre comment les mots se désolidarisent des significations et des objets auxquels ils étaient attachés ouvrant ainsi la voie à de possibles objectivations nouvelles. 
MoTS CLÉS

autoconfrontation croisée - clinique de l'activité- reprise- hétérogénéité

\section{RESUMEN}

La vida de las palabras en el análisis del trabajo. Los métodos de análisis del trabajo que dan cuenta de la clínica de la actividad comprometen a los profesionales a una confrontación con las huellas que deja la filmación de las acciones realizadas. Este dispositivo resulta evolutivo en la medida que, potencialmente, abre caminos para pensar/decir/actuar la actividad cotidiana en forma diferente. En el marco de este artículo, los datos extraídos de una intervención se apoyan en esta orientación metodológíca desarrollada con los actores de la Validación de lo Adquirido a partir de la Experiencia. A partir del estudio del proceso lingüístico creativo se abre una pista que permite describir con precisión el impacto que produce retomar las palabras del profesional por parte del investigador. Se utiliza el concepto de heterogeneidad de Bajtín, para comprender cómo las palabras se desolidarizan de las significaciones y de los objetos a los cuales estaban asociados, abriendo de esta forma a posibles nuevas objetivaciones.

Palabras Clave

Autoconfrontación cruzada, clínica de la actividad, reanudación, heterogeneidad.

\section{RÉFÉRENCEMENT}

Henry, M., \& Bournel Bosson, M. (2008). La vie des mots en analyse du travail. @ctivités, 5 (2) pp. xx-xxx, http://www.activites.org/v5n2/v5n2.pdf

Article soumis le 5 novembre 2007, accepté pour publication le 8 juillet 2008 . 